CVIA

ORIGINAL ARTICLE

pISSN 2508-707X / elSSN 2508-7088 https://doi.org/10.22468/cvia.2018.00171 CVIA 2018;2(4):194-201

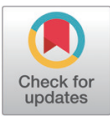

\section{Factors Determining Inadequate Stress Response to Adenosine Triphosphate in Perfusion Cardiovascular Magnetic Resonance}

\author{
Andrew KC Cheng, Jubi YL Li, Sonia HY Lam, Stephen CW Cheung \\ Department of Radiology, Queen Mary Hospital, Hong Kong, China
}

\begin{abstract}
Objective: Adenosine triphosphate (ATP) is an established pharmacological agent for stress cardiovascular magnetic resonance (CMR) in the Asia Pacific region. Inadequate cardiac stress in a CMR study may lead to false-negative results. The aim of the present study is to identify the proportion of patients with inadequate stress using a $140 \mathrm{mcg} / \mathrm{kg} / \mathrm{min}$ protocol and the factors for inadequate stress in ATP stress CMR.
\end{abstract}

Materials and Methods: A retrospective analysis from January to December 2017 was performed. In total, 150 patients underwent ATP stress perfusion CMR at 1.5 tesla. All patients received ATP infusion of $140 \mathrm{mcg} / \mathrm{kg} / \mathrm{min}$ for $4 \mathrm{~min}$. Adequate stress was defined as two or more of the following criteria: 1) positive splenic switch off sign, 2) increased heart rate $\geq 10 \mathrm{bpm}$, and 3) drop in systolic blood pressure $\geq 10 \mathrm{~mm} \mathrm{Hg}$. Multivariable logistic regression analysis with forward and backward stepwise selection was used to identify predictors of inadequate response to ATP. Basic demographic variables with $p$-value $\leq 0.2$ were examined for inclusion in the model. A p-value less than 0.05 was considered significant.

Received: July 31, 2018

Revised: September 16, 2018

Accepted: September 27, 2018

Corresponding author

Andrew KC Cheng, MD

Department of Radiology,

Queen Mary Hospital,

102 Pok Fu Lam Road

Hong Kong, China

Tel: 852-2255-3300

Fax: 852-2817-5496

E-mail: acheng1404@gmail.com
Results: Fifty-six (37\%) patients did not demonstrate adequate cardiac stress response. Non-responders to ATP more often had atrial fibrillation, higher indexed left ventricular end systolic volume, and lower ejection fraction. Multivariable logistic regression analysis showed that low body weight and male gender were consistent independent predictors of inadequate stress in all models.

Conclusion: Inadequate stress occurred in $37 \%$ of subjects with ATP infusion of $140 \mathrm{mcg} / \mathrm{kg} /$ min. Low body weight and male gender are independent predictors of inadequate response to ATP. Higher infusion rates of ATP should be considered in patients with inadequate stress.

Key words Magnetic resonance imaging · Adenosine triphosphate - Stress test.

\section{INTRODUCTION}

Stress cardiovascular magnetic resonance (CMR) is a highly accurate, safe, and non-invasive method for diagnosis of coronary artery disease $(\mathrm{CAD})$ and has been incorporated into guidelines for diagnosis of obstructive CAD [1-6]. Stress CMR is commonly performed using vasodilatory pharmacological stress agents such as adenosine or adenosine triphosphate (ATP) $[7,8]$. These adenosine-based compounds generate systemic vasodilatation and reflex sympatho excitation with a consequent decease in systolic blood pressure (SBP), increase in heart rate (HR),

(c) This is an Open Access article distributed under the terms of the Creative Commons Attribution Non-Commercial License (https://creativecommons.org/licenses/bync/4.0) which permits unrestricted non-commercial use, distribution, and reproduction in any medium, provided the original work is properly cited. and modest increase in rate-pressure product $[9,10]$.

As potent coronary vasodilators, adenosine and ATP cause an up to four-fold increase in myocardial blood flow in areas supplied by normal coronary arteries. With gadolinium-based contrast agents, reduced flow gives rise to regional perfusion defects in the presence of coronary stenosis [7].

However, some patients fail to demonstrate adequate hemodynamic changes (increased HR and decreased SBP), indicating suboptimal physiological stress. These non-responders are at risk of false-negative findings on stress CMR perfusion and suboptimal clinical management.

The splenic switch off (SSO) sign has been recently demonstrated. The short axis image orientation, where most myocardial perfusion magnetic resonance (MR) images are acquired, 
usually includes the spleen. There is marked attenuation of splenic perfusion signal in the stress images compared with the rest of the images. The SSO sign has recently been shown to gauge the adequacy of CMR stress [11].

ATP infusion rates in some centers have been set at $160 \mathrm{mcg} /$ $\mathrm{kg} / \mathrm{min}$ for $5 \mathrm{~min}[10,12-20]$. The purpose of this retrospective study is to identify the proportion of patients with inadequate stress using a lower dose $140 \mathrm{mcg} / \mathrm{kg} / \mathrm{min}$ protocol and to determine the factors related to inadequate stress in ATP stress CMR.

\section{MATERIALS AND METHODS}

This study was conducted under approval by the Institutional Review Board (UW 18-317). This was a retrospective noninterventional analysis of the database of our institution from 1st January 2017 to 31st December 2017. The inclusion criteria were patients with known or suspected CAD referred for stress CMR imaging for clinical purposes. Exclusion criteria were patients with asthma, unstable angina, or acute myocardial infarction within two weeks of the study or second or third degree atrioventricular block. A 12-lead electrocardiogram was performed before the CMR scan. All patients were asked to abstain from caffeine for 24 hours prior to the scan. However, patients who reported caffeine intake within the last 24 hours were still included in the study. Written consent are obtained from participants.

\section{Adenosine triphosphate infusion protocol}

All patients initially had an ATP injection with an infusion rate of $140 \mathrm{mcg} / \mathrm{kg} / \mathrm{min}$ for $4 \mathrm{~min}$ through an antecubital vein using a syringe pump. Hemodynamic status (i.e., HR, BP, and oxygen saturation) was determined at $0,1,2$, and 3 min after ATP infusion. If, after $4 \mathrm{~min}$ of continuous infusion at the rate of $140 \mathrm{mcg} / \mathrm{kg} / \mathrm{min}$, the hemodynamic response to ATP was inadequate (increase in HR less than $10 \mathrm{bpm}$ or decrease in systolic BP less than $10 \mathrm{~mm} \mathrm{Hg}$ ), the clinician would decide either to proceed to scan or increase the infusion rate to $170 \mathrm{mcg} / \mathrm{kg} / \mathrm{min}$.

\section{CMR protocol}

All CMR examinations were performed with subjects in a supine position on a 1.5 MR Tesla instrument (Aera, Siemens AG, Erlangen, Germany) with a 32-element phased array coil. During the last minute of ATP infusion, a gadolinium-based contrast agent was administered intravenously (injection rate of 4 $\mathrm{mL} / \mathrm{s}$, followed by a $20 \mathrm{~mL}$ saline flush at the same flow rate). Perfusion imaging was performed every cardiac cycle during the first pass, using a T1 weighted fast gradient echo sequence [dynamic trufi echo time (TE) $0.98 \mathrm{~ms}$, repetition time (TR) $177 \mathrm{~ms}$, saturation recovery time $110 \mathrm{~ms}$, voxel size $2.3 \times 2.3 \times 8$ $\mathrm{mm}$, flip angle $50^{\circ}$. Three short axis slices, positioned from the base to the apex of the left ventricle, were obtained. The same imaging sequence was repeated 10 minutes later without ATP to obtain perfusion images at rest. For assessment of left ventricular function, steady-state free precession (SSFP) cine images (TE/TR $1.28 / 40.17 \mathrm{~ms}$, voxel size $1.2 \times 1.2 \times 6 \mathrm{~mm}$, flip angle $62^{\circ}$ ) were acquired in three long axis views and a short axis stack to obtain coverage of the entire left ventricle (LV). Analysis of LV function was performed with syngo.via (Siemens AG) using the short axis SSFP images. The following left ventricular parameters were determined: end diastolic volume (EDV), end systolic volume (ESV), ejection fraction (EF), and LV mass.

\section{Splenic perfusion analysis}

A recently proposed marker of adequate stress is splenic blood flow attenuation during stress perfusion (i.e., spleen appears hypointense in signal) [11]. Splenic first-pass perfusion was analyzed by a fellow radiologist using syngo.via (Siemens AG). Splenic regions of interest were placed on stress and rest perfusion images to generate curves showing mean splenic signal intensity (SI, arbitrary units) changes over time. Filling of the LV was defined as time zero to ensure the synchronous comparison of rest and stress scans. Peak splenic perfusion SI was estimated as the numerical difference between baseline SI and maximal SI during the splenic first-pass perfusion. Splenic intensity ratio (SIR) was calculated as peak splenic perfusion SI during stress divided by peak splenic perfusion SI during rest. The SSO sign was defined as positive if SIR was less than 0.4 (Figs. 1, 2, and 3).

\section{Adequate hemodynamic response}

Adequate hemodynamic response was defined as an increase in $\mathrm{HR} \geq 10 \mathrm{bpm}$ and a decrease in systolic $\mathrm{BP} \geq 10 \mathrm{~mm}$ Hg during the ATP infusion period.

\section{Adequate stress}

Adequate stress was defined as the presence of two or more of the following criteria: 1) positive SSO sign, 2) HR increase $\geq 10$ bpm, and 3) systolic BP decrease $\geq 10 \mathrm{~mm}$ Hg during ATP infusion. Inadequate stress was defined as $\leq 1$ of the above criteria.

\section{Statistical analyses}

Continuous variables were expressed as mean \pm standard deviation and were assessed by independent Student's t-test. Categorical variables were expressed as count and percentage. Comparisons of categorical variables between groups were performed with Pearson's chi-square test. Multivariable logistic regression analysis was performed for variables with $p$-value $\leq 0.2$. We used multivariable logistic regression analysis with forward and backward stepwise selection and $95 \%$ confidence intervals to identify potential predictors of inadequate response. 

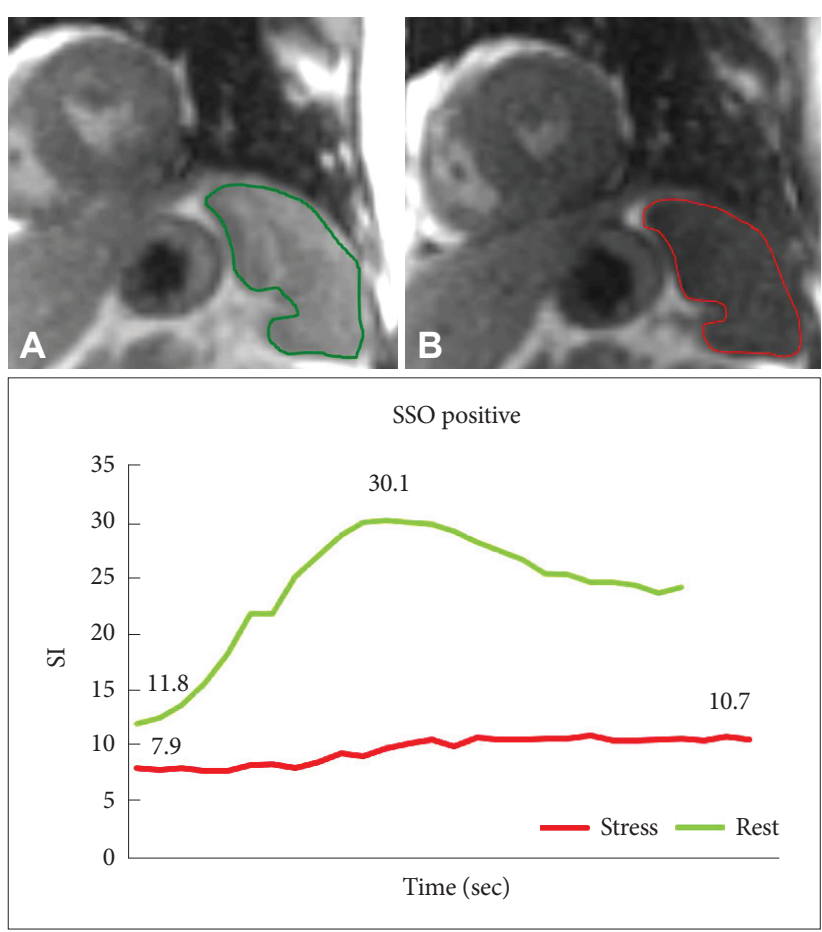

Fig. 1. Representative stress and resting splenic first-pass gadolinium perfusion, showing SI curves with positive SSO sign. SI curves represent splenic perfusion $\mathrm{SI}$ (y-axis, arbitrary units) versus time (xaxis). The maximum and minimum spleen SI values are as indicated. Splenic intensity ratio $=0.15$. (A) Splenic regions of interests on perfusion images are outlined in green during rest. (B) Splenic regions of interests on perfusion images are outlined in red during stress. SSO: splenic switch off, Sl: signal intensity.

A p-value less than 0.05 was considered significant, and twotailed p-values were used for all statistics. Statistical analyses were performed using statistical software (SPSS version 25.0 for Windows, IBM Corp., Armonk, NY, USA).

\section{RESULTS}

\section{Patient characteristics}

Data were collected from 150 consecutive subjects with a mean age of $61 \pm 15$ years (range: 15-90 years), of whom 81 (54\%) were men. See Table 1 for patient characteristics of those with and without adequate stress. See Table 2 for hemodynamic parameters.

Nineteen (12\%) of 150 patients showed stress-induced myocardial ischemia on perfusion CMR (Table 3). Of the $131 \mathrm{pa}-$ tients without stress-induced myocardial ischemia, 81 (61\%) achieved adequate stress. Of the total 150 patients, eight underwent angiographic coronary angiogram within one year after the CMR study. Of these eight patients, four (50\%) were found to have severe CAD ( $>70 \%$ stenosis). Two of the four patients who were diagnosed angiographically with severe $\mathrm{CAD}$ had negative CMR examination. One of the two (50\%) of them had inadequate stress.
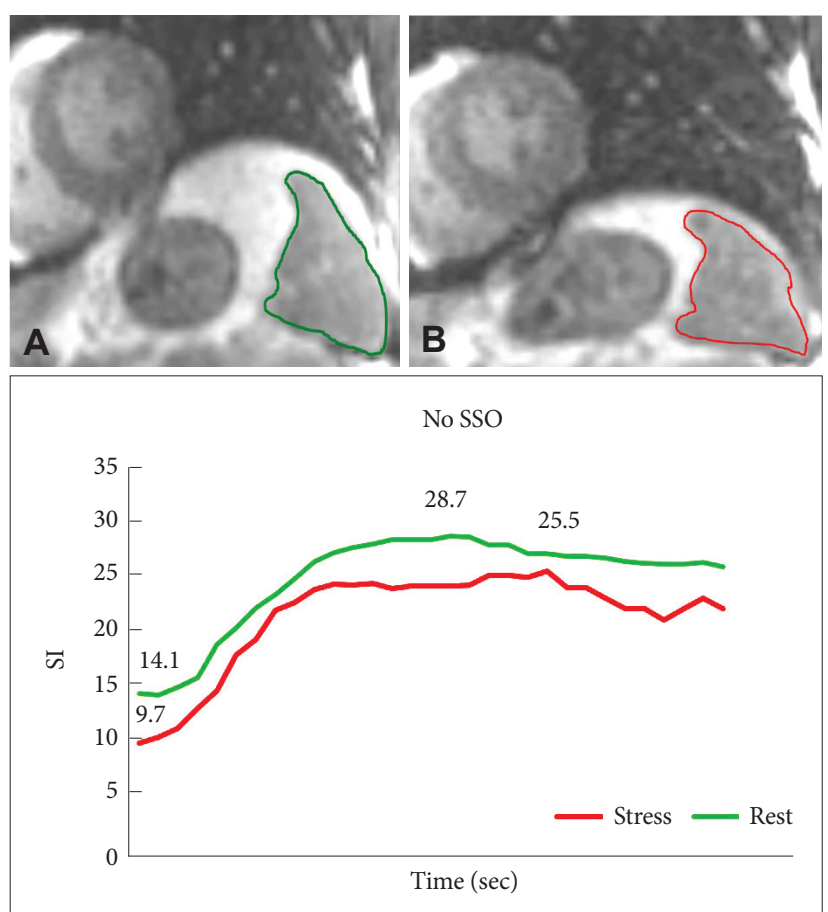

Fig. 2. Representative stress and resting splenic first-pass gadolinium perfusion, showing SI curves with no SSO sign. SI curves represent splenic perfusion $\mathrm{SI}(\mathrm{y}$-axis, arbitrary units) versus time (x-axis). The maximum and minimum spleen SI values are as indicated. Splenic intensity ratio=1.0. (A) Splenic regions of interests on perfusion images are outlined in green during rest. (B) Splenic regions of interests on perfusion images are outlined in red during stress. SSO: splenic switch off, SI: signal intensity.
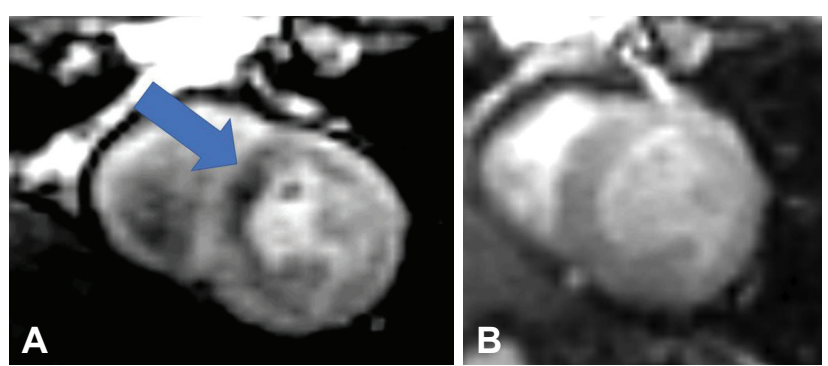

Fig. 3. A representative example from a CMR perfusion scan during high-dose adenosine stress. The patient was an 88-year-old man with history of dyslipidemia. Note the marked perfusion defect in the septum and anterior wall (arrow) in the left anterior descending artery territory $(A)$. The resting perfusion scan $(B)$ is normal and shows homogenous enhancement in all myocardial regions.

\section{Adequate hemodynamic response and adequate stress}

Ninety-four of 150 patients (62\%) experienced adequate stress at the ATP infusion rate of $140 \mathrm{mcg} / \mathrm{kg} / \mathrm{min}$. In addition, 84 of the $94(89 \%)$ patients had adequate hemodynamic responses (i.e., increase in $\mathrm{HR} \geq 10 \mathrm{bpm}$ and decrease in systolic $\mathrm{BP} \geq 10$ $\mathrm{mm} \mathrm{Hg}$ ).

Five of the 56 patients (9\%) with inadequate hemodynamic responses received a higher infusion rate of ATP of $170 \mathrm{mcg} /$ $\mathrm{kg} / \mathrm{min}$. Two of these five patients ( $40 \%$ ) achieved adequate he- 
modynamic response.

\section{Splenic switch off sign}

SIR ranged from 0.15 to 1.6 ; the mean SIR was $0.66 \pm 0.29$. The SSO sign was identified in 29 (19\%) of 150 patients. Within the adequate stress group, 27 (28\%) of the 94 patients demonstrated a positive SSO sign. The mean SIR for the adequate stress

Table 1. Patient characteristics of study population

\begin{tabular}{|c|c|c|c|}
\hline & $\begin{array}{c}\text { Adequate } \\
\text { stress group } \\
(\mathrm{n}=94)\end{array}$ & $\begin{array}{c}\text { Inadequate } \\
\text { stress group } \\
\quad(\mathrm{n}=56)\end{array}$ & $\mathrm{p}$ value \\
\hline Age (yr) & $60 \pm 15$ & $62 \pm 16$ & 0.500 \\
\hline Male & $46(49)$ & $35(62)$ & 0.100 \\
\hline Height $(\mathrm{cm})$ & $161.2 \pm 9.7$ & $163 \pm 8.9$ & 0.260 \\
\hline Weight (kg) & $67 \pm 15.8$ & $63.3 \pm 12.1$ & 0.130 \\
\hline BSA & $1.7 \pm 0.2$ & $1.68 \pm 0.2$ & 0.280 \\
\hline Caffeine $<24$ prior to scan & $4(4)$ & $4(7)$ & 0.450 \\
\hline Hypertension & $57(60)$ & $31(55)$ & 0.530 \\
\hline Diabetes & $28(29)$ & $16(28)$ & 0.870 \\
\hline Hyperlipidemia & $46(48)$ & $23(41)$ & 0.350 \\
\hline Smoking & $12(12)$ & $11(19)$ & 0.250 \\
\hline End-diastolic volume index $(\mathrm{mL})$ & $73 \pm 15$ & $81 \pm 27$ & 0.050 \\
\hline End-systolic volume index (mL) & $27 \pm 11$ & $36 \pm 26$ & 0.019 \\
\hline Ejection fraction (\%) & $63 \pm 8$ & $57 \pm 13$ & 0.008 \\
\hline Mass index $\left(\mathrm{gram} / \mathrm{m}^{2}\right)$ & $69 \pm 16$ & $72 \pm 23$ & 0.380 \\
\hline Beta-blocker & $41(43)$ & $25(44)$ & 0.900 \\
\hline Ca-channel blocker & $32(34)$ & $21(37)$ & 0.660 \\
\hline ACE-inhibitor & $23(24)$ & $15(26)$ & 0.750 \\
\hline Statin & $63(67)$ & $39(69)$ & 0.730 \\
\hline Aspirin & $47(50)$ & $22(39)$ & 0.200 \\
\hline Digoxin & $1(1)$ & $3(5)$ & 0.100 \\
\hline Glomerular filtration rate & $85 \pm 20$ & $81 \pm 19$ & 0.250 \\
\hline Atrial fibrillation & $1(1)$ & $5(8)$ & 0.017 \\
\hline Ectopic & $6(6)$ & $6(10)$ & 0.344 \\
\hline $\mathrm{HR}$ at rest (bpm) & $67.5 \pm 14.1$ & $70.4 \pm 12.9$ & 0.200 \\
\hline $\mathrm{SBP}$ at rest $(\mathrm{mm} \mathrm{Hg})$ & $146 \pm 19$ & $140 \pm 21$ & 0.070 \\
\hline $\mathrm{DBP}$ at rest $(\mathrm{mm} \mathrm{Hg})$ & $84 \pm 12$ & $83 \pm 11$ & 0.500 \\
\hline ATP infusion time (min) & $4.7 \pm 0.4$ & $5 \pm 0.8$ & 0.020 \\
\hline
\end{tabular}

Data are presented as mean \pm standard deviation or count (\%). BSA: body surface area, HR: heart rate, SBP: systolic blood pressure, DBP: diastolic blood pressure, ATP: adenosine triphosphate group was $0.59 \pm 0.27$. For the inadequate stress group, 2 (3\%) of the 56 patients demonstrated a positive SSO sign. The mean SIR for the inadequate stress group was $0.77 \pm 0.2$ (Table 4 ).

\section{Hemodynamic response and splenic switch off sign}

Seventeen of 84 patients (21\%) with adequate hemodynamic response demonstrated the SSO sign. Twelve of 66 patients (18\%) without adequate hemodynamic response demonstrated the SSO sign (Table 5).

\section{Adverse events}

There were no deaths, myocardial infarctions, or episodes of pulmonary edema as a result of the ATP stress.

\section{Predictors of inadequate stress from ATP infusion of $140 \mathrm{mcg} / \mathrm{kg} / \mathrm{min}$}

Patients who showed inadequate stress in response to ATP infusion more often had atrial fibrillation, significantly higher indexed left ventricular ESV, and lower EF. Variables that showed a trend toward significance $(\mathrm{p} \leq 0.2)$ were gender, weight, SBP at rest, $\mathrm{HR}$ at rest, atrial fibrillation, aspirin use, indexed EDV, indexed ESV, and LV EF.

CMR volumes and LV EF were not included since they are likely to be unavailable prior to stress. Digoxin and caffeine intake within 24 hours were not included, as only four and eight patients, respectively, had been prescribed digoxin or consumed caffeine. Only six patients had atrial fibrillation and were not included. The potential predictors of inadequate stress included for adjustment were gender, weight, SBP at rest, and HR at rest. By multivariable logistic regression analysis, the predictors of inadequate stress are low body weight $(\mathrm{p}=0.02)$ and male gender $(\mathrm{p}=0.019)$ (Table 6).

Table 3. Summarizing the subsequent management for patients diagnosed with ischemia changes

\begin{tabular}{lc}
\hline \multicolumn{1}{c}{ CMR with ischemia } & Total=19 \\
\hline With invasive PCI performed within 1 year after CMR & 4 \\
Conservative or medical treatment & 12 \\
Further work up with CT coronary angiogram & 2 \\
Defaulted Follow up & 1 \\
\hline
\end{tabular}

CMR: cardiovascular magnetic resonance, PCI: percutaneous coronary intervention

Table 2. Hemodynamic parameters at rest and during adenosine stress

\begin{tabular}{|c|c|c|c|c|c|c|}
\hline & \multicolumn{3}{|c|}{ Adequate stress group $(\mathrm{n}=94)$} & \multicolumn{3}{|c|}{ Inadequate stress group $(n=56)$} \\
\hline & Rest & Peak & $\mathrm{p}$ value & Rest & Peak & $\mathrm{p}$ value \\
\hline HR (bpm) & $67 \pm 14$ & $85 \pm 14$ & $<0.001$ & $70 \pm 12$ & $78 \pm 14$ & 0.002 \\
\hline SBP (mm Hg) & $146 \pm 19$ & $119 \pm 17$ & $<0.001$ & $140 \pm 21$ & $122 \pm 21$ & $<0.001$ \\
\hline DBP (mm Hg) & $84 \pm 12$ & $70 \pm 10$ & $<0.001$ & $83 \pm 11$ & $72 \pm 12$ & $<0.001$ \\
\hline Rate pressure product & $9928 \pm 2644$ & $10233 \pm 2512$ & 0.42 & $9918 \pm 2652$ & $9715 \pm 2920$ & 0.7 \\
\hline
\end{tabular}

Data are presented as mean \pm standard deviation. SBP: systolic blood pressure, DBP: diastolic blood pressure, HR: heart rate 
Table 4. Hemodynamic response, SSO, and adequate stress to standard dose adenosine

\begin{tabular}{lcccc}
\hline & Adequate stress group $(\mathrm{n}=94)$ & Inadequate stress group $(\mathrm{n}=56)$ & $\mathrm{p}$ value & Total $(\mathrm{n}=150)$ \\
\hline Adequate haemodynamic response & $84(89)$ & $0(0)$ & $<0.001$ & $84(56)$ \\
SIR & $0.59 \pm 0.27$ & $0.77 \pm 0.2$ & $<0.001$ & $0.66 \pm 0.29$ \\
SSO positive $($ SIR $<0.4)$ & $27(28)$ & $2(3)$ & $<0.001$ & $29(19)$ \\
\hline
\end{tabular}

Data are presented as mean \pm standard deviation or count (\%). SSO: splenic switch off, SIR: splenic intensity ratio

Table 5. Comparison of SSO and hemodynamic response rate $(p=$ $0.75)$

\begin{tabular}{lccc}
\hline & $\begin{array}{c}\text { Adequate } \\
\text { hemodynamic } \\
\text { response }\end{array}$ & $\begin{array}{c}\text { Inadequate } \\
\text { hemodynamic } \\
\text { response }\end{array}$ & Total \\
\hline SSO & 17 & 12 & 29 \\
No SSO & 67 & 54 & 121 \\
Total & 84 & 66 & 150 \\
\hline
\end{tabular}

SSO: splenic switch off

Table 6. Logistic regression model with gender, weight, SBP at rest, $\mathrm{HR}$ at rest, atrial fibrillation as variables

\begin{tabular}{lccccc}
\hline Variable & Coefficient & $\begin{array}{c}\text { Standard } \\
\text { error }\end{array}$ & Sig. & $\begin{array}{c}\text { Odds } \\
\text { ratio }\end{array}$ & $\begin{array}{c}\text { 95\% confidence } \\
\text { interval }\end{array}$ \\
\hline Male & 1.016 & 0.432 & 0.019 & 2.762 & $1.185-6.438$ \\
Weight & -0.036 & 0.016 & 0.020 & 0.964 & $0.935-0.994$ \\
SBP at rest & -0.015 & 0.009 & 0.096 & 0.985 & $0.967-1.003$ \\
HR at rest & 0.021 & 0.013 & 0.104 & 1.021 & $0.996-1.048$ \\
\hline
\end{tabular}

Predictor for inadequate stress is low body weight $(\mathrm{p}=0.02)$ and male gender $(\mathrm{p}=0.019)$. SBP: systolic blood pressure, HR: heart rate

\section{DISCUSSION}

In our study, 56 of 150 patients (37\%) did not demonstrate adequate cardiac stress response with the ATP infusion rate of $140 \mathrm{mcg} / \mathrm{kg} / \mathrm{min}$. In addition, we found that low body weight and male gender were independent predictors of inadequate stress response.

ATP is typically infused at a rate of $140 \mathrm{mcg} / \mathrm{kg} / \mathrm{min}$ [21] or $160 \mathrm{mcg} / \mathrm{kg} / \mathrm{min}$ [10]. There are currently limited data on their differences in terms of inadequate cardiac stress, adverse reactions, and false-negative rates. To our knowledge, ours is the first study with a lower ATP infusion rate of $140 \mathrm{mcg} / \mathrm{kg} / \mathrm{min}$ to determine the proportion of inadequate cardiac stress responses and the independent predictors of inadequate cardiac stress. We demonstrated that up to $63 \%$ of patients can achieve adequate cardiac stress with the infusion rate of $140 \mathrm{mcg} / \mathrm{kg} / \mathrm{min}$.

Without adjustment, the inadequate stress group had significantly higher indexed LV ESV, lower EF, and more atrial fibrillation in our study. Karamitsos et al. [4] used adenosine for perfusion CMR and concurred with our findings that the inadequate stress group had significantly higher indexed LV ESV and lower EF. The proportion of inadequate stress in our study (37\%) was higher compared with that reported by Karamitsos et al. [4], who demonstrated that $18 \%$ of patients did not achieve adequate hemodynamic stress. This is probably attributable to our protocol of relatively low ATP infusion rate $(140 \mathrm{mcg} / \mathrm{kg} /$ $\mathrm{min}$ ) for $4 \mathrm{~min}$. From the literature, ATP can be infused intravenously at $160 \mathrm{mcg} / \mathrm{kg} / \mathrm{min}$ for $5 \mathrm{~min}[10,12-20,22]$. Karamitsos et al. [4] found that age and reduced EF were the predictors of inadequate response. We did not replicate this result. These findings may be attributed to different sample sizes, stress agents, ethnic groups, and most importantly, the criteria for defining adequate stress. In contrast to Karamitsos et al. [4], we had a greater sample size of patients, with a total of 150 subjects, which would bring a greater level of confidence. Second, our data are predominantly from a Chinese population, while the study by Karamitsos et al. [4] is based on a Caucasian population. Third, Karamitsos et al. [4] defined inadequate stress solely by hemodynamic response (HR increase $<10 \mathrm{bpm}$ or SBP decrease $<10$ $\mathrm{mm} \mathrm{Hg}$ ).

Hosking et al. [23] demonstrated that lack of SSO is prevalent, easily measurable, and has the potential to improve upon hemodynamic criteria as a marker of adenosine under stress in CMR perfusion scans. Hosking et al. [23] suggested that further work combining SSO with hemodynamic response should be performed to minimize false-negative rates further. In our study, we combined the assessment of hemodynamic response and SSO sign as the criteria of adequate cardiac stress. This combined assessment would be more objective and may minimize false-negative rates.

ATP has been shown to be safe and to elicit adequate stress with effects similar to those of adenosine $[13,17,22]$. There are also fewer side effects and lower costs with ATP infusion $[14,15,20]$. ATP has been documented to demonstrate the SSO sign, which is not present with the use of other pharmacological agents such as regadenoson [12,22]. One disadvantage for ATP infusion is the relatively long infusion time needed to achieve a pharmacological stress response compared with adenosine [22].

Male gender was shown to be an independent predictor of inadequate stress in our models. This phenomenon is supported by Miyagawa et al. [10], who demonstrated that male gender was associated with significantly lower adverse effects of ATP infusion in thallium-201 myocardial tomography. This phenomenon is not well understood, but our postulation is that there could be a gender difference in cardiac adenosine receptors. Low body weight was shown to be another independent 
predictor of inadequate stress, which is likely explained by the dose-dependent response to ATP. The total amount of ATP given is dependent on the patient's body weight. This finding is supported by Ylldırım Poyraz et al. [24], who demonstrated that obese patients suffered more from the side effects of adenosine during myocardial perfusion SPECT/CT imaging. From our receiver-operative characteristic curve, the cut-off of low body weight was defined as $\leq 65.8 \mathrm{~kg}$ with a sensitivity of $51.8 \%$ and specificity of $51.1 \%$ [area under the curve (AUC): $0.55 \pm 0.04$ ].

The SSO is a new sign with a clear potential clinical application for assessment of stress adequacy during adenosine myocardial stress perfusion MR imaging. Manisty et al. [11] demonstrated that failed SSO occurred in more than one-third of proven false-negative adenosine perfusion examinations, almost four times more commonly than in examinations with true negative results. The SSO can either be measured quantitatively or assessed visually. In our study, we quantitatively measured the SIR. The SSO sign is defined as positive if SIR is less than 0.4. This threshold of SIR for the SSO sign is based on Hosking et al. [23] (sensitivity $=82.5 \%$, specificity $=92.3 \%, \mathrm{AUC}=0.91$ ).

HR and mean arterial pressure changes are known to be poor predictors of myocardial blood flow and coronary vascular resistance changes. It may be misleading to determine stress adequacy from hemodynamic responses alone [25]. Recent studies have shown that the hyperemic and hemodynamic responses to adenosine correlate poorly [25]; therefore, SSO is only weakly associated with hemodynamic response. In our study, we observed a poor correlation between hemodynamic response and the SSO sign $(\mathrm{p}=0.75)$. From the literature [26], there was a good correlation of myocardial hyperemic response to adenosine with the splanchnic vasoconstrictor response. Therefore, the SSO sign is thought to be a better indicator of adequate adenosine stress than is the hemodynamic response. In our study, the SSO sign was only identified in 29 (19\%) of 150 patients. One explanation is the relatively low ATP infusion rate (140 $\mathrm{mcg} / \mathrm{kg} / \mathrm{min}$ for $4 \mathrm{~min}$ ) in our study compared to Miyagawa et al. [10], where ATP was infused intravenously at $160 \mathrm{mcg} / \mathrm{kg} /$ $\min$ for $5 \mathrm{~min}$. It may also be explained by the semi-quantitative measure of SIR with the cut-off at 0.4. Indeed, we did demonstrate that the SI ratio was lower in the adequately stressed patients than in those not adequately stressed.

Inadequate stress CMR may lead to false-negative results and suboptimal clinical management. One future strategy is to measure real time splenic flow directly during adenosine-induced stress before contrast administration, which would ensure that perfusion images are acquired only when an adequate stress response is achieved. From Liu et al. [27], the delta T1 of the spleen can facilitate optimization of stress responses before gadolinium first-pass perfusion CMR. This enables prediction of the visual SSO sign without the need for gadolinium. It potentially guides adenosine dosage up-titrations before contrast injection, thereby improving the quality of stress response before first-pass perfusion imaging [27]. One disadvantage of this methodology is the extra time required for T1-mapping. Further research is warranted to determine whether T1-mapping decreases the number of false-negative perfusion scans.

Stress CMR has a reported false-negative rate of 5-16\% [2830]. In our study, 131 of 150 patients had normal perfusion CMR findings, but 50 of the 131 patients (38\%) had inadequate stress (Table 7). This group of patients is subjected to the potential risk of false-negative results. Inadequate adenosine stress response is the most common cause of false-negative perfusion scans [30]. Initiating higher dose adenosine protocols may reduce falsenegative rates on myocardial perfusion studies by up to $33 \%$, according to Manisty et al. [11]. In our study, 56 patients (37\%) had an inadequate response to the ATP infusion at $140 \mathrm{mcg} / \mathrm{kg} /$ $\mathrm{min}$. Of note, only five of these 56 patients received an increased ATP dose of $170 \mathrm{mcg} / \mathrm{kg} / \mathrm{min}$ for further cardiac stress. Two out of the five patients (40\%) who received an increased ATP dose subsequently achieved an adequate hemodynamic stress. The implementation rate of the high dose protocol was conceivably low in our study. Clinicians should vigilantly implement the high dose protocol if they observe an inadequate stress response.

Karamitsos et al. [4] demonstrated that a high dose adenosine protocol (up to $210 \mathrm{mcg} / \mathrm{kg} / \mathrm{min}$ ) is well tolerated, safe, and results in adequate hemodynamic response in nearly all patients. The infusion rate of ATP that causes maximal coronary vasodilation has not been determined [10]. In our study, the high dose ATP protocol was $170 \mathrm{mcg} / \mathrm{kg} / \mathrm{min}$, with a maximum of $210 \mathrm{mcg} / \mathrm{kg} / \mathrm{min}$. Whether the ATP doses of $170 \mathrm{mcg} / \mathrm{kg} / \mathrm{min}$ and $210 \mathrm{mcg} / \mathrm{kg} / \mathrm{min}$ would reduce the rate of inadequate stress warrants further research.

Watkins et al. [31] compared the diagnostic accuracy of perfusion CMR versus fractional flow reserve. In a per-patient analysis, the sensitivity and specificity of perfusion CMR for the detection of functionally significant CAD were $95 \%$ and $91 \%$, respectively, with respective positive and negative predictive values of $97 \%$ and $84 \%$. Stress perfusion CMR can detect functionally significant $\mathrm{CAD}$ with excellent diagnostic accuracy [12].

The strength of our study is the large number of patients involved. We also used a new objective measure of adequate stress, the SSO sign, to help determine stress. Male sex and low body

Table 7. Comparison of CMR results and stress adequacy

\begin{tabular}{lccr}
\hline & $\begin{array}{c}\text { Normal CMR } \\
\text { perfusion }\end{array}$ & $\begin{array}{c}\text { CMR } \\
\text { with ischemia }\end{array}$ & Total \\
\hline Adequate stress & 81 & 13 & 94 \\
Inadequate stress & 50 & 6 & 56 \\
Total & 131 & 19 & 150 \\
\hline
\end{tabular}

CMR: cardiovascular magnetic resonance 
weight as independent predictors can routinely be determined prior to stress CMR, allowing one to better identify the potential risk of inadequate stress.

This study has limitations. Our study is a single-center retrospective study; therefore, further research is required to determine if results can be replicated. Second, our study population involved a predominantly Chinese population, which may render our study results less generalizable. Third, ATP-related side effects were not available for review. We believe that our study provides further data on the predictors of inadequate stress in stress CMR. The use of ATP is prevalent in the Asia Pacific region [16-19], and our data are likely relevant and similar to those of other Asia Pacific CMR centers. Further research is required to determine if the same predictors can be replicated in other populations. Correlating the predictors with catheter coronary angiography data and fractional flow reserve would be useful to determine the impact of inadequate stress on false-negative rates in stress CMR.

In conclusion, our study indicates that independent predictors of inadequate ATP stress in stress CMR are low body weight and male sex. In addition, the proportion of inadequate stress was $37 \%$ with an ATP infusion rate of $140 \mathrm{mcg} / \mathrm{kg} / \mathrm{min}$. Clinicians should consider higher infusion rates in patients with inadequate stress.

\section{Conflicts of Interest}

The authors declare that they have no conflict of interest.

\section{Acknowledgments}

The authors would like to thank Dr. Ng Ming-Yen for supporting this work. Dr. Ng Ming-Yen contributed in expert advice and data analysis.

\section{REFERENCES}

1. Greenwood JP, Maredia N, Younger JF, Brown JM, Nixon J, Everett CC, et al. Cardiovascular magnetic resonance and single-photon emission computed tomography for diagnosis of coronary heart disease (CE-MARC): a prospective trial. Lancet 2012;379:453-460.

2. Schwitter J, Wacker CM, Wilke N, Al-Saadi N, Sauer E, Huettle K, et al. MR-IMPACT II: Magnetic Resonance Imaging for Myocardial Perfusion Assessment in Coronary artery disease Trial: perfusion-cardiac magnetic resonance vs. single-photon emission computed tomography for the detection of coronary artery disease: a comparative multicentre, multivendor trial. Eur Heart J 2013;34:775-781.

3. Bettencourt N, Chiribiri A, Schuster A, Ferreira N, Sampaio F, Duarte R, et al. Cardiac magnetic resonance myocardial perfusion imaging for detection of functionally significant obstructive coronary artery disease: a prospective study. Int J Cardiol 2013;168:765-773.

4. Karamitsos TD, Ntusi NA, Francis JM, Holloway CJ, Myerson SG, Neubauer S. Feasibility and safety of high-dose adenosine perfusion cardiovascular magnetic resonance. J Cardiovasc Magn Reson 2010;12:66.

5. Chen J, Einstein AJ, Fazel R, Krumholz HM, Wang Y, Ross JS, et al. Cumulative exposure to ionizing radiation from diagnostic and therapeutic cardiac imaging procedures: a population-based analysis. J Am Coll Cardiol 2010;56:702-711.

6. Walker S, Girardin F, McKenna C, Ball SG, Nixon J, Plein S, et al. Costeffectiveness of cardiovascular magnetic resonance in the diagnosis of coronary heart disease: an economic evaluation using data from the CE-
MARC study. Heart 2013;99:873-881.

7. Gerber BL, Raman SV, Nayak K, Epstein FH, Ferreira P, Axel L, et al. Myocardial first-pass perfusion cardiovascular magnetic resonance: history, theory, and current state of the art. J Cardiovasc Magn Reson 2008; 10:18.

8. Christiansen JP, Karamitsos TD, Myerson SG, Francis JM, Neubauer S. Stress perfusion imaging using cardiovascular magnetic resonance: a review. Heart Lung Circ 2010;19:697-705.

9. Wilson RF, Wyche K, Christensen BV, Zimmer S, Laxson DD. Effects of adenosine on human coronary arterial circulation. Circulation 1990;82: 1595-1606.

10. Miyagawa M, Kumano S, Sekiya M, Watanabe K, Akutzu H, Imachi T, et al. Thallium-201 myocardial tomography with intravenous infusion of adenosine triphosphate in diagnosis of coronary artery disease. J Am Coll Cardiol 1995;26:1196-1201.

11. Manisty C, Ripley DP, Herrey AS, Captur G, Wong TC, Petersen SE, et al. Splenic switch-off: a tool to assess stress adequacy in adenosine perfusion cardiac MR imaging. Radiology 2015;276:732-740.

12. Yamada A, Ishida M, Kitagawa K, Sakuma H. Assessment of myocardial ischemia using stress perfusion cardiovascular magnetic resonance. Cardiovasc Imaging Asia 2018;2:65-75

13. Belhassen B, Pelleg A. Electrophysiologic effects of adenosine triphosphate and adenosine on the mammalian heart: clinical and experimental aspects. J Am Coll Cardiol 1984;4:414-424.

14. Cerqueira MD, Verani MS, Schwaiger M, Heo J, Iskandrian AS. Safety profile of adenosine stress perfusion imaging: results from the Adenoscan Multicenter Trial Registry. J Am Coll Cardiol 1994;23:384-389.

15. Coma-Canella I, Palazuelos J, Bravo N, García Velloso MJ. Myocardial perfusion imaging with adenosine triphosphate predicts the rate of cardiovascular events. J Nucl Cardiol 2006;13:316-323.

16. Watanabe K, Sekiya M, Ikeda S, Miyagawa M, Kinoshita M, Kumano S. Comparison of adenosine triphosphate and dipyridamole in diagnosis by thallium-201 myocardial scintigraphy. J Nucl Med 1997;38:577-581.

17. Chun KA, Lee J, Lee SW, Ahn BC, Ha JH, Cho IH, et al. Direct comparison of adenosine and adenosine 5 '-triphosphate as pharmacologic stress agents in conjunction with Tl-201 SPECT: Hemodynamic response, myocardial tracer uptake, and size of perfusion defects in the same subjects. J Nucl Cardiol 2006;13:621-628.

18. Yao Z, Zhu H, Li W, Chen C, Wang H, Shi L, et al. Adenosine triphosphate stress myocardial perfusion imaging for risk stratification of patients aged 70 years and older with suspected coronary artery disease. J Nucl Cardiol 2017;24:429-433.

19. Tomiyama Y, Manabe O, Oyama-Manabe N, Naya M, Sugimori H, Hirata K, et al. Quantification of myocardial blood flow with dynamic perfusion 3.0 Tesla MRI: Validation with (15) O-water PET. J Magn Reson Imaging 2015;42:754-762.

20. Bravo N, Giménez M, Mejía S, García-Velloso MJ, Coma-Canella I. Prognostic value of myocardial perfusion imaging with adenosine triphosphate. J Nucl Cardiol 2002;9:395-401.

21. Kitagawa K, Sakuma H, Nagata M, Okuda S, Hirano M, Tanimoto A, et al. Diagnostic accuracy of stress myocardial perfusion MRI and late gadolinium-enhanced MRI for detecting flow-limiting coronary artery disease: a multicenter study. Eur Radiol 2008;18:2808-2816.

22. Bartolomé Leal P, Quilez Larragan A, Garcia Baizán A, Millor Muruzábal M, Pueyo Villoslada JC, Bastarrika Alemañ G; Pamplona/ES. Adenosine and adenosine triphosphate (ATP) have a similar stress effect in patients undergoing stress/rest perfusion cardiac magnetic resonance examinations. B-0983. Poster session presented at: European Congress Radiology 2017; 2017 Mar 1-5; Vienna, Austria.

23. Hosking A, Koulouroudias M, Zemrak F, Moon JC, Rossi A, Lee A, et al. Evaluation of splenic switch off in a tertiary imaging centre: validation and assessment of utility. Eur Heart J Cardiovasc Imaging 2017;18:12161221.

24. Yıldırım Poyraz N, Özdemir E, Poyraz BM, Kandemir Z, Keskin M, Türkölmez Ş. Predictors and diagnostic significance of the adenosine re- 
lated side effects on myocardial perfusion SPECT/CT imaging. Mol Imaging Radionucl Ther 2014;23:89-95.

25. Mishra RK, Dorbala S, Logsetty G, Hassan A, Heinonen T, Schelbert HR, et al. Quantitative relation between hemodynamic changes during intravenous adenosine infusion and the magnitude of coronary hyperemia: implications for myocardial perfusion imaging. J Am Coll Cardiol 2005;45: 553-558.

26. Norlén K. Central and regional haemodynamics during controlled hypotension produced by adenosine, sodium nitroprusside and nitroglycerin. Studies in the pig. Br J Anaesth 1988;61:186-193.

27. Liu A, Wijesurendra RS, Ariga R, Mahmod M, Levelt E, Greiser A, et al. Splenic T1-mapping: a novel quantitative method for assessing adenosine stress adequacy for cardiovascular magnetic resonance. J Cardiovasc Magn Reson 2017;19:1.

28. Hamon M, Fau G, Née G, Ehtisham J, Morello R, Hamon M. Meta-analysis of the diagnostic performance of stress perfusion cardiovascular magnetic resonance for detection of coronary artery disease. J Cardiovasc
Magn Reson 2010;12:29.

29. Jaarsma C, Leiner T, Bekkers SC, Crijns HJ, Wildberger JE, Nagel E, et al. Diagnostic performance of noninvasive myocardial perfusion imaging using single-photon emission computed tomography, cardiac magnetic resonance, and positron emission tomography imaging for the detection of obstructive coronary artery disease: a meta-analysis. J Am Coll Cardiol 2012;59:1719-1728.

30. Kidambi A, Sourbron S, Maredia N, Motwani M, Brown JM, Nixon J, et al. Factors associated with false-negative cardiovascular magnetic resonance perfusion studies: a clinical evaluation of magnetic resonance imaging in coronary artery disease (CE-MARC) substudy. J Magn Reson Imaging 2016;43:566-573.

31. Watkins S, McGeoch R, Lyne J, Steedman T, Good R, McLaughlin MJ, et al. Validation of magnetic resonance myocardial perfusion imaging with fractional flow reserve for the detection of significant coronary heart disease. Circulation 2009;120:2207-2213. 